\title{
Evaluation of the Triphasic Pulse Stimulation in Eliminating Facial Nerve Stimulation in Cochlear Implant Recipients
}

\author{
Mohamed Zakaria Ezzeldin Abdelhamed* \\ School of Advanced Education Research and Accreditation, Saudi Arabia
}

Submission: December 11, 2018; Published: January 22, 2019

*Corresponding author: Mohamed Zakaria Ezzeldin Abdelhamed, School of Advanced Education Research and Accreditation, Saudi Arabia

Abstract

The objectives of this study were to determine if the triphasic stimulation pulses strategy can eliminate facial nerve stimulation (FNS) in cochlear implant (CI) users, and to determine the required change(s) in fitting parameters and patient performance with triphasic strategy.

Method: Subjects were five CI users with FNS that could not be eliminated by other methods. They were 4 males and one female. All fitting parameters (THR, MCL, Compression, Sensitivity, Maplow, etc.) and patient performance (Aided Thresholds and SRT) were re-measured with triphasic strategy and compared to the biphasic strategy.

Results: Triphasic pulse stimulation strategy eliminated FNS in all subjects and showed better patient performance than biphasic stimulation pulses strategy, but required modifications in fitting parameters to achieve patient satisfaction.

Conclusion: Triphasic stimulation is highly effective way to eliminate FNS in the CI users.

Keywords: Cochlear implant; Fitting; Biphasic stimulation; Triphasic stimulation, Facial nerve stimulation

Abbreviations: CI: Cochlear Implant HL: Hearing Loss; SNHL: Sensorineural Hearing Loss; FDA: Food and Drug Administration; dB: Decibel; Hz: Hertz; THR: Threshold; MCL: Most Comfortable Level; AGC: Automatic Gain Control; IIDR: Instantaneous Input Dynamic Range; FNS: Facial Nerve Stimulation; SSD: Single Sided Deafness; IP2: Incomplete partition Type 2; PTA: Pure Tone Threshold; SRT: Speech Reception Threshold

\section{Introduction}

Cochlear implant (CI) is a medical electronic device that was invented to help patients who have sensorineural hearing loss to restore normal hearing or near to normal hearing via electric stimulation of the auditory nerve endings inside the human cochlea. CI is a partially implanted solution which consists of internal and external part. The Internal part is named the implant and the external part is the audio processor which is worn over the ear, receives sounds, analyses them, and send it to the internal implant. As Mudry \& Mills [1] mention in 1961, the first CI was implanted by William House and John Doyle in Los Angeles, California. Since then, the number of CI is tremendously increasing across the world. From 30 June, 2012-30 June, 2013, approximately 50,000 cochlear implants were sold. Given the world population of about seven billion, this means that roughly seven cochlear implants per million individuals are sold each year. Approximately 30,000 of these 50,000 cochlear implants were received by children. Some of these children received bilateral implants, which means that 25,000 individual children received unilateral or bilateral cochlear implants. Children and adults who are deaf or severely hard-of hearing are main recipients of CI. As of December 2012, approximately 324,200 registered devices have been implanted worldwide. In the United States, roughly 58,000 devices have been implanted in adults and 38,000 in children as Kamal [2] mentioned. After the internal part (the implant) is implanted in the patient head and the wound is totally healed, the implant is switch on and fitting sessions begin to program the audio processor. After variable umber of programming sessions over a period 3-6 months, the vast majority of CI recipients are able to hear normal or near normal.

\section{Hearing and Hearing Loss Types}

Hearing is one of the sensory systems of the human body. It is part of the communication skills that human has. It is curtail for every body for everyday life. Any hearing problems or disability may affect the quality of life. Ear is the main organ that responsible for hearing sensation. It consists of three main parts outer ear, middle ear and inner ear. The sound waves is picked up by the pinna and directed towards the external ear canal, the 
sound waves hit the ear drum at the end of the external ear canal and causes the ear drum to vibrate. The ossicles which are at the middle ear and attached from one side to the ear drum start also to vibrate. Through their shape, physical properties, and the way they connect with each other amplify the vibrations. The ossicles are the malleus, incus and stapes. The malleus is directly attached to the ear drum and the stapes is attached to the cochlea through a window which is named oval window. The vibrations finally propagate inside the cochlea which is filled with fluid. When this fluid vibrates, it causes displacements at the basilar membrane where the organ of corti is. Organ of corti has hair cells that respond to any displacement in the basilar membrane and start firing electrochemical pulses that are picked up by the auditory nerve. Figure 1 illustrates the ear anatomy. Normal hearing covers a frequency range of sounds between $20 \mathrm{~Hz}$ and $20,000 \mathrm{~Hz}$. The majority of speech sounds are within a frequency range of between 100 and $8000 \mathrm{~Hz}$. The human ear is most sensitive to frequencies around 1000-3500Hz. Hearing loss can be classified into main four types: conductive, sensorineural (SNHL), mixed, and neural. There are also several causes of hearing loss in each categorical type.

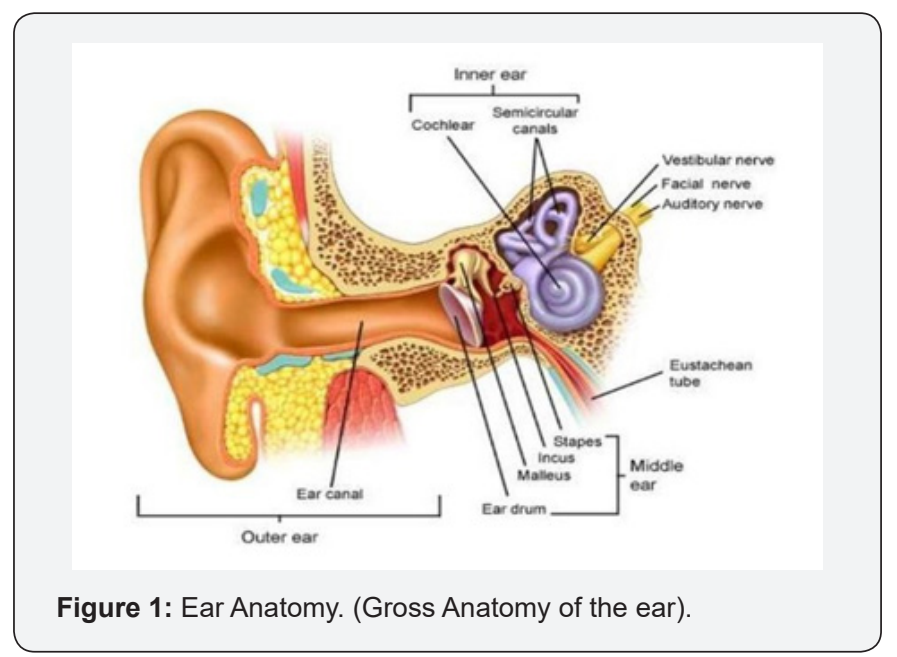

\section{Conductive hearing loss}

In such type of hearing loss, sound vibrations are not reaching the cochlea with all its power. There is a pathology that is blocking or delaying the sound vibration to reach the cochlea, leading to a reduction in ear sensation to sound. This pathology can be fluid, blood, pus, or adhesions in the middle ear. The middle ear pathology can be caused by infection or trauma. Ear trauma can also leads to ossicular discontinuity. Excessive ear wax in the external auditory canal can also lead to conductive hearing loss. Conductive hearing loss can be cured by medications, surgeries or using hearing aids or combinations of them.

\section{Sensorineural hearing loss}

This kind of hearing loss happens when there is pathology in the cochlear sensory cells (outer and inner hair cells) or its vascular supply (stria vascularis). In such case, the cochlear sensory are not responding in a normal way to the vibrations of the basilar membrane and fail to transduce such vibrations to electrical pulses that can pass through the auditory nerve. This leads to hearing loss of varying degrees. This kind of hearing loss cannot be surgically or medically treated as the damage of the hair cells is usually irreversible. Hearing aids are the usual line of management for SNHL. However, in cases of severe degree of SNHL, hearing aids cannot restore hearing. In such cases CI is the best line of management. The most common cause of SNHL in children is congenital, usually of genetic etiology but it can be acquired due to insults to the cochlea in the early neonatal period. Examples of such insults are neonatal hypoxia, neonatal infection and neonatal hyperbilirubinemia. Other causes of SNHL include ototoxic drugs, severe head trauma, presbycusis, and chronic exposure to load sounds.

\section{Mixed hearing loss}

This kind of hearing loss occurs when there are two components of conductive and SNHL that causes hearing loss. It results from problems in both the inner and outer or middle ear. Treatment options may include medication, surgery, hearing aids or hearing implants.

\section{Neural hearing loss}

This kind of hearing loss occurs when the pathology is in the auditory nerve or higher auditory nervous system (retrocochlear). Neural hearing loss is usually difficult to treat.

\section{Degree of hearing loss}

The hearing loss is also classified according to its degree into mild, moderate, severe, and to profound hearing loss; (Figure 2). The degrees of hearing loss can be determined just by looking into the patient's audiogram. An audiogram is a graph illustrating a person's usable hearing and the amount of hearing loss that an individual has for each ear. The audiogram illustrates the degree of hearing sensitivity and hence the degree of hearing loss at each sound frequency from $250 \mathrm{~Hz}$ to $8000 \mathrm{~Hz}$, as a standard. CI is indicated when there is severe, severe to profound or profound degree of hearing loss. When the degree of hearing loss is less than severe, hearing aids are beneficial. As mention in MED-EL that cochlear implant (CI) is a medical electronic device that is indicated for patients who have severe to profound hearing loss. $\mathrm{CI}$ is the only medical device capable of replacing a sense. It works by bypassing non- functioning parts of the inner ear and providing electrical stimulation directly to nerve fibres in the cochlea. CI consists of internal part (Implant; Figure 3) and external part (Audio processor; Figure 4 The internal part consists of internal coil, magnet, electronic package, and electrode. While the external part consists of control unit, coil, magnet, coil cable, battery pack and battery pack cover. As Kamal [2] mentioned there are mainly three cochlear implants manufactures; MED-EL based in Innsbruck-Austria, Cochlear based in Australia, and Advanced Bionics based in United States. 


\section{Global Journal of Otolaryngology}

Cochlear Corporation device uses 22 electrodes spaced along its array; Advanced Bionics implant has a 16 element array, while the MED-EL electrode has 12 pairs of electrodes (each pair sharing the same position along the array).

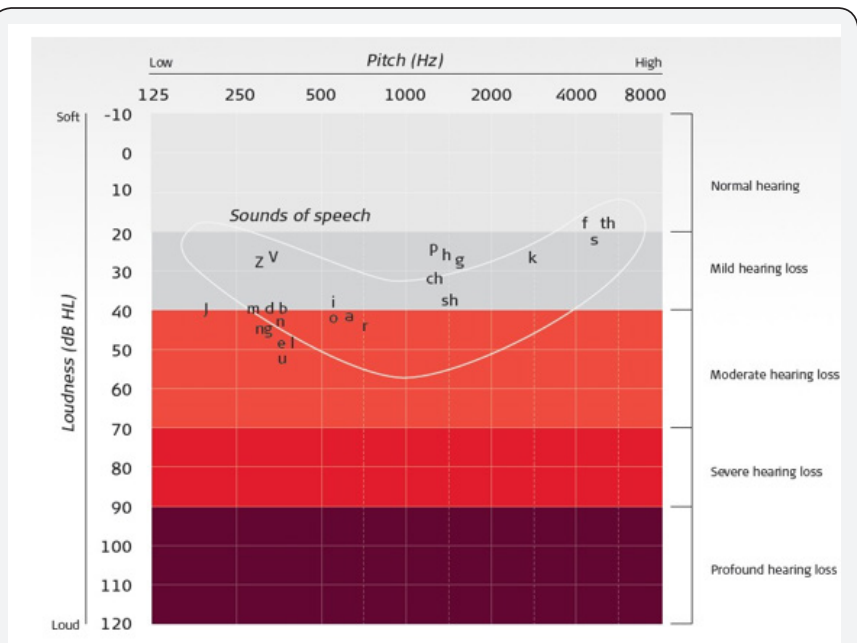

Figure 2: Degrees of hearing loss (MED-EL, 2018)

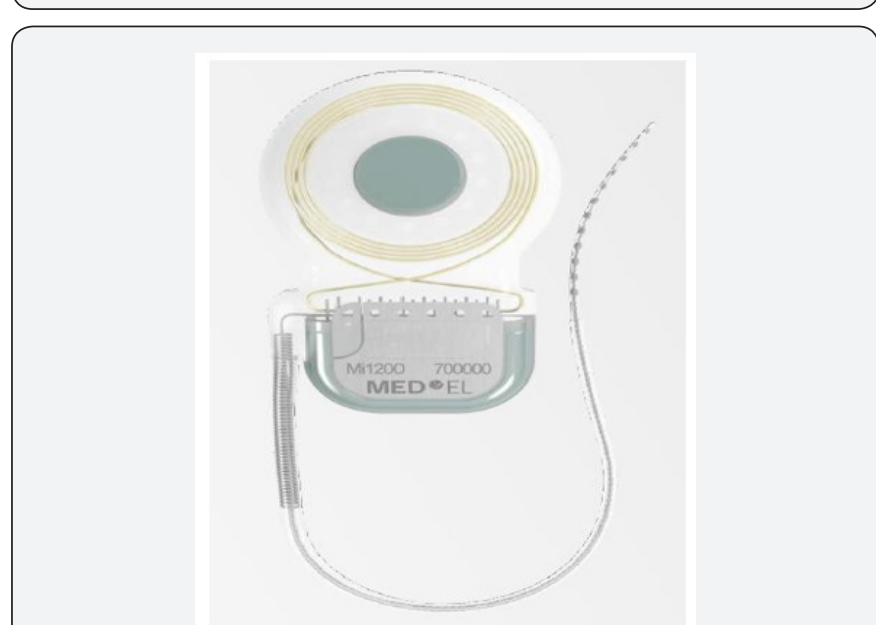

Figure 3: The MEDEL SYNCHRONY internal implanted part of the cochlear implant (MED-EL, 2018).

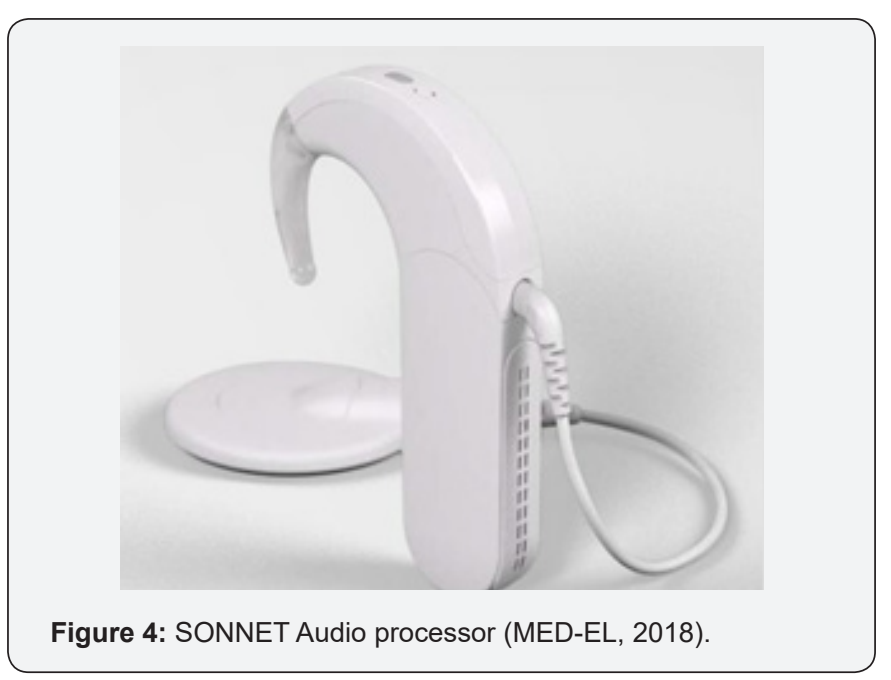

\section{How does the CI work?}

Figure 5 illustrates how CI works. Sound is collected by the microphone embedded in the external audio processor which processes the sound signal through some complicated analog and digital algorithms and then sends the signal to the internal part through radio frequency waves through the processor coil. The internal part under the skin receives and demodulates the signal and sends it to the electrode that was implanted inside the cochlea. Each electrode consists of numbers of contacts; each contact is responsible of some range of frequencies and stimulates the neural cells in some way to match the incoming sound as Kamal [2] mentioned, the electrical signals cause activity of the fibers of the auditory nerve, and the brain interprets this as sound.

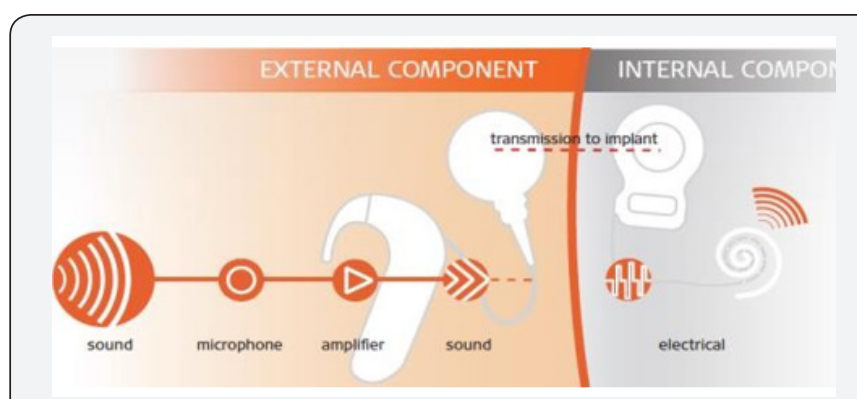

Figure 5: Diagram illustrating how cochlear implant works (MED-EL, 2018).

\section{Cochlear implant sound coding}

A sound coding strategy describes in detail the way this is carried out. As MED-EL mentions that Sound coding strategies can vary in how efficiently aspects of the sound signal are transmitted, and what priority is given to these aspects. These variables of sound coding strategies have a direct effect on the quality of the hearing experience. There are two types of coding:

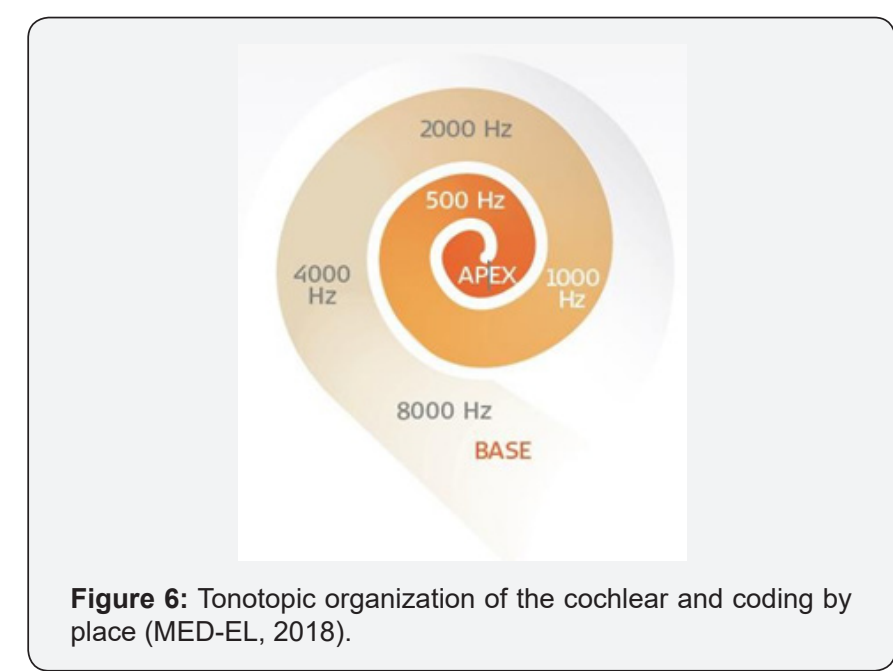

Coding by place: It corresponds to the tonotopic arrangement of the cochlea. Like the keys of a piano, the cochlea is arranged in order of frequency and in normal hearing, each place along 
the cochlea responds best to a certain frequency. The base of the cochlea responds best to high frequencies, whereas the apex responds best to low frequencies. The pattern of activity in both the auditory nerve and the brain match this arrangement. Figure 6 shows such tonotopic organization of the cochlea. The audioprocessor of the $\mathrm{CI}$ analyses the incoming sound and spilt it into different frequencies and send each frequency component to its corresponding place in the implanted electrode that contacts a corresponding place of the auditory nerve within the cochlea. By this way, CI preserves the normal tonotopic arrangement of the cochlea.

Code by rate: The second fundamental mechanism of sound coding in normal hearing is coding by rate. In the cochlea the hair cells are responsible for converting the movements of the membrane in the cochlea into electrical impulses. Due to the way the hair cells respond to this movement, the firing pattern of the auditory nerve activity closely corresponds to the timing pattern of the sound signal. This process works well in normal hearing for frequencies up to $\sim 1 \mathrm{kHz}$. As frequency increases, the efficiency of phase locking decreases, until about $4-5 \mathrm{kHz}$, where phase locking no longer operates MED-EL [3]. Figure 7 shows how the cochlea preserves the rate of the incoming sound. To a large extent, CI mimics the cochlear in preserving the rate of the incoming sound. Since stimulating multiple electrodes at the same time can give an unpredictable loudness percept because of channel interactions (addition of stimulus voltage fields) most current commercial coding strategies use sequential stimulation. The rate of pulse stimulation to an electrode depends on processing strategy. The slowest pulse rates in use are 200 pulses/s (pps). A pulse rate of around 800 pps is common to several strategies, while higher rates of up to 5000 pps can be used in some recent strategies. With pulsatile stimulation within these ranges of rate, the percept is not of a burst of pulses, but rather as a continuous signal.

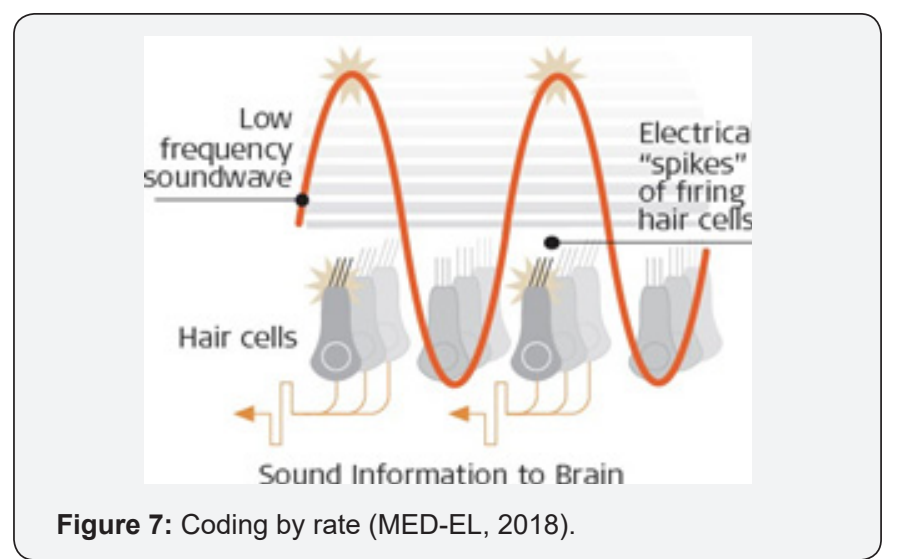

\section{Candidacy of Cochlear Implants}

Cochlear implant candidacy criteria have evolved dramatically since multichannel implants were first approved for adult use by the FDA in 1985 and in 1990 for the pediatric population. Initially, only individuals with bilateral profound sensorineural hearing loss with no open set speech recognition were considered candidates for cochlear implantation. Over time, however, these criteria have become less stringent and individuals with greater amounts of residual hearing are now being implanted Gifford \& René [4]. Currently, the candidacy of cochlear implant is usually divided according to the age (pediatric or adult).

\section{Adult candidacy of CI}

Figure 8 displays the evolution of audiometric criteria of CI adult candidacy over the years to the most current encompassing region of audiometric thresholds for moderate sloping to profound sensorineural hearing loss. Initially, CI was restricted to adult deaf patients with profound degree of SNHL (i.e., hearing loss more than $90 \mathrm{~dB}$ HL) Gifford \& René [4]. After the success of $\mathrm{CI}$ in such category, CI was recommended for patient with only severe degree of hearing loss (from $70 \mathrm{~dB}$ HL to $90 \mathrm{~dB} \mathrm{HL}$ ), for whom hearing aids did not help. CI had excellent results in such degree of hearing loss. Finally, CI is currently indicated for patients with only moderate degree of SNHL at the lower frequencies and severe to profound degree at higher frequencies. In such case, the external audio-processor works as hearing aid in the lower frequencies and as the processor for $\mathrm{CI}$ in the high frequencies. Such scenario is referred to as electroacoustic stimulation or hybrid stimulation.

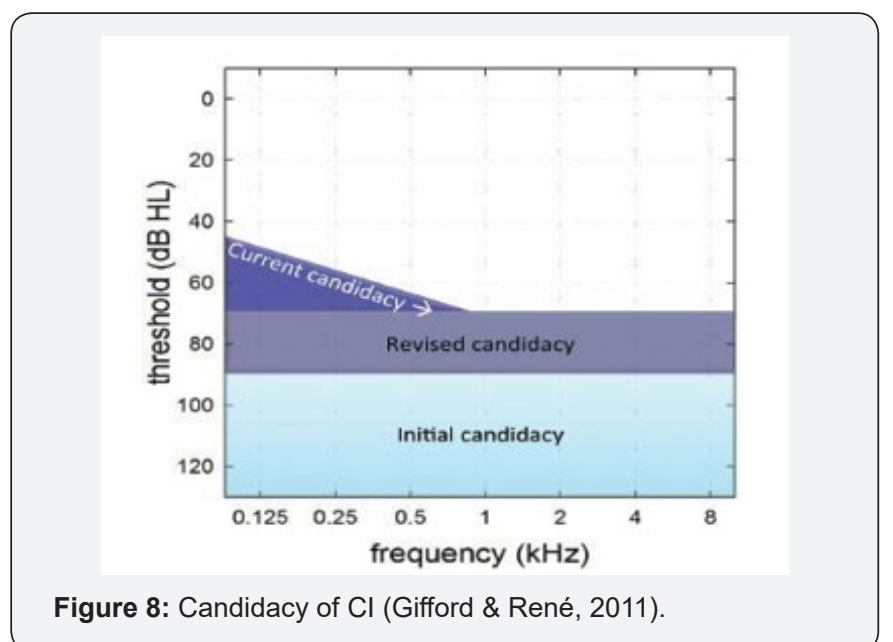

\section{Pediatric candidacy of CI}

Children are the main recipients of CI. Currently CI is permitted for children with severe to profound hearing loss and of the age of one year and older. A trial period of hearing aid for 3 to 6 months is required before the CI surgery. In addition, CT scan and MRI to check the status of the cochlea and auditory nerve is also required.

\section{Cochlear Implant Programming}

Two to four weeks after the CI surgery, the audio-processor has not have to be programmed to deliver the optimal electrical impulses to the auditory nerve. The ultimate goal of device 
programming is to adjust it so that it can effectively convert acoustic input into a usable electric signal for each electrode stimulated. With proper programming, the device converts the acoustic signals (speech and non-speech signals) into electrical pulses and delivers them to the auditory nerve terminals within the cochlea, and to maintain the specific parameters of the acoustic signals similar to what the normal cochlea does to a large extent. Proper programming is critical to achieve the ultimate goals of CI which are normal speech perception and hence development of oral language in cases of children. The most crucial aspect of programming a cochlear implant is to establish the lowest and highest usable stimulation level for each electrode in the array, and this is a common feature of all cochlear implant manufacturers as Kamal [2] mentioned. Therefore, two basic psychophysical measures need to be obtained on each intracochlear electrode: electrical thresholds (THR or T level), defined as the softest level at which a patient is stimulated $100 \%$ of the time, and most comfortable loudness levels (MCL, C or M levels), defined as the loudest sound a patient can listen to comfortably for a sustained period of time Shapiro \& Bradham [5]. The range in $\mathrm{dB}$ between the lowest intensity that can be coded within the auditory nerve and highest intensity represents the electrical dynamic range. Such electrical dynamic range is very limited when is compared to the acoustic dynamic range of speech (around $100 \mathrm{~dB}$ ). The challenge of the CI audioprocessor is to compress the wider acoustic dynamic range to the much smaller electrical dynamic range. This is achieved by specific characters within the processor which are the Automatic Gain Control (AGC) and the instantaneous input dynamic range (IIDR). Such compression is crucial for speech perception.

\section{Most common Fitting Parameters}

As Kamal [2] mentions in his study, the most important fitting parameters are listed below. It must be noted that these parameters are somehow strategy and device dependent. Some parameters are presented in different devices under different names. Almost all of these parameters are used in the fitting of all three manufacturers' implants.

a. MCL, M level, or $\mathrm{C}$ level is the most comfortable stimulation level that the $\mathrm{CI}$ recipient can tolerate

b. TRL or T level is the softest stimulation level that the CI recipient can detect.

c. Stimulation current: It presents the current amplitude that is given to each electrode; it is calculated differently from one manufacturer to another.

d. Phase duration: The duration of applying every single pulse, it is named also pulse width.

e. Charge Unit: It is the product of stimulation current and the phase duration, actually this is what CI recipient hears.

f. Pulse rate: The number of pulses that is given to the cochlea every second.

g. Frequency bands: dedicated frequency range presented by each electrode.

h. Coding strategy: The sound coding protocol applied on the signal inside the speech processor.

i. Maplaw: It controls the progress of amplitude growth function and its compression characteristics.

j. Sensitivity: It defines the sensitivity of the speech processor microphones. It is the range that the microphone can detect.

k. Directionality: Several microphone modes which control the incoming signal to eliminate noises and mimic natural hearing.

l. Compliance level: This is the maximum power the implant can give at each channel.

\section{Cochlear implant surgery and complications}

The surgery is performed under general anesthesia and takes approximately 1 to 3 hours. The procedure is considered a routine surgery with low risk. In this part, it is presented the general steps of CI operation, risks and complications.

General steps of CI surgery (American cochlear implant alliance):

a. Skin opening: The surgeon will make a $4-6 \mathrm{~cm}$ incision behind the ear in a double flap technique.

b. Mastiodoctomy: The surgeon opens the mastoid bone leading to the middle ear space; it is the best and most direct way to access the middle ear.

c. Posterior Tympanotomy: Also known as facial recess, Opening a window from the mastoid to the middle ear between the facial nerve and the chorda tympani.

d. Round window opening: The surgeon then makes an opening in the round window of the cochlea or near to it (cochleostomy).

e. CI placement: The surgeon then places the receiver/ stimulator, the electronic portion of the device attached to the electrode array, under the skin behind the ear and secures it in place.

f. Electrode insertion: The implant electrode array is then inserted into the cochlea.

g. Skin closure: The incision is then closed and a head dressing is applied to protect the incision.

\section{Risks and side effects of surgery (American Cochlear Implant Alliance)}

Cochlear implantation surgery risks are the same or lower than other common ear surgeries. Rarely the following can occur: 
a. Bleeding and/or swelling at the incision site.

b. Infection in the area of the implant

c. Ringing (tinnitus) in the implanted ear.

d. Dizziness or vertigo (typically resolves within a few days after surgery).

e. Change in taste/dry mouth (typically resolves within a few weeks or months after surgery).

f. Numbness around the incision site

g. Injury to the facial nerve.

Pain tolerance is different for everyone, but in general the pain is mild-to-moderate and can be controlled with oral pain medications, if needed, for a few days. During the posterior tympanotomy, the surgeon is very close to the facial nerve and corda tympani that why it is very important in the operating room to have facial nerve monitoring machine. This machine is monitoring if the surgeon accidentally touches the facial nerve while drilling or not and will give alarm if this happened. If the surgeon does damage the facial nerve, the patient will have a facial nerve paralysis and will affect half of the patients face macules. Also if any damage occurs to the corda tympani, the taste sensation of the patient will be badly affected. These effects are permanent and irreversible. Therefore, facial nerve monitor machine is considered to be mandatory in the cochlear implant surgery.

\section{Modes of Cochlear Implant Stimulation}

Stimulation mode refers to the electrical current flow, that is, the location of the reference electrode relative to the active stimulating electrode. Monopolar stimulation refers to a remote ground reference (outside of the cochlea). Figure 9 shows this monopolar stimulation in MEDEL implant, where the ground electrode lies in the implant under the skin while the actual active electrodes are implanted within the cochlea. In bipolar stimulation, both the active and ground electrode is within the cochlea. For the Cochlear manufacture, the device can be programmed in both monopolar and bipolar stimulation mode while the Advanced Bionics and MED- EL devices can be programmed in a monopolar mode only. Typically monopolar stimulation is the preferred mode, as this mode may extend battery life, allowing for a more consistent thresholds and threshold value for adjacent electrodes due to a wider current spread. In addition, this mode is more suitable than bipolar in the interpolation of THR and MCL levels in populations in whom obtaining psychophysical measure on every electrode implanted is not feasible Shapiro, Bradham and William [5]. In all the three CI manufacturers, the CI stimulates the auditory nerve with series of short biphasic electrical pulses. The pulses are biphasic because the net current through the tissue should be zero to avoid unwanted long-term electrochemical effects as Kamal [2] mentioned in his study. Figure 10 shows the biphasic pulse stimulation in MED-EL CI. Recently, MED-EL introduced another way of stimulation with several different pulse shapes and different inter pulse gaps. They are called triphasic pulse and precision triphasic pulses (Figure 11).

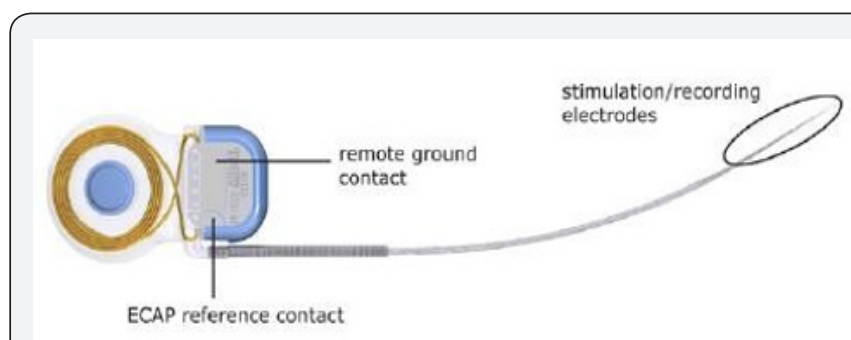

Figure 9: Mono-polar stimulation in MED-EL Implant (MED-EL, 2018).

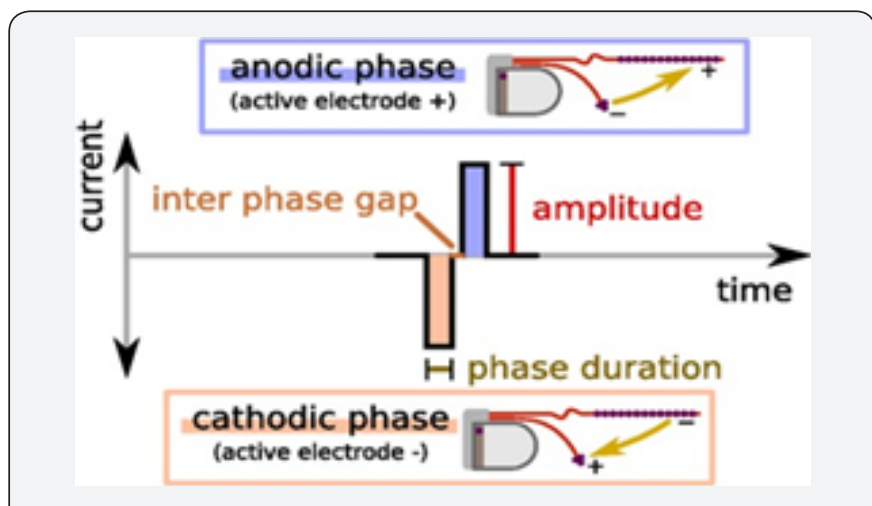

Figure 10: Biphasic pulse stimulation (MED-EL, 2018).

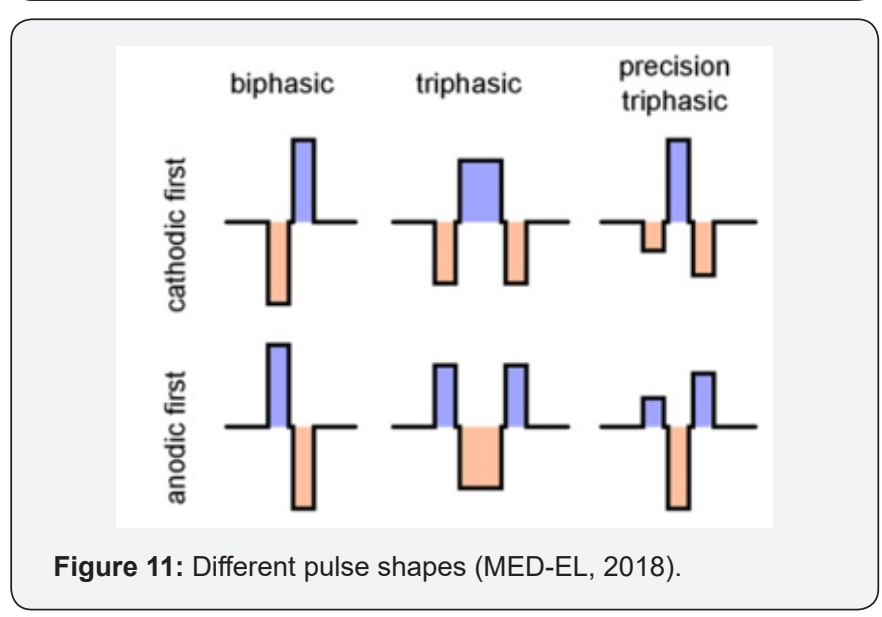

\section{Facial Nerve Stimulation in CI}

Post-implantation facial nerve stimulation is one of the wellknown and most frequent complications of the cochlear implant procedure. Some conditions, such as otosclerosis and cochlear malformations, as well as high stimulation levels that may be necessary in patients with long auditory deprivation expose patients to a higher risk of developing post-implant facial nerve stimulation. Facial nerve stimulation following CI occurs when the electrodes inside the cochlea are activated and the facial nerve gets wrongly stimulated too. This non- auditory stimulation is not desired in the CI process. Facial nerve stimulation following CI occurs in $6.5 \%$ CI users. There is no difference in rate of occurrence of the facial nerve stimulation among the three $\mathrm{CI}$ 
manufacturers. Some anatomical factors contribute to such unwanted facial nerve stimulation. These factors include that the implanted electrode pass across the facial recess, making them very close to the facial nerve. The electrical current may spread from the $\mathrm{CI}$ electrodes and stimulates the facial nerve. This occurs more frequently because the $\mathrm{CI}$ electrode is inserted inside the cochlea and the facial nerve (labyrinthine portion part) is passing near the cochlea near the basal and med turn of the cochlea. So sometimes its gets wrongly stimulated. It was also hypothesized that the array of electrodes could erode the bony layer between the scala tympani and facial nerve, making the electrical current passing through CI electrode closest to the facial nerve Berrettini et al. [6]. Other factors responsible for facial nerve stimulation are low impedance pathway at the modiolar base, high stimulation levels necessary to stimulate hypoplasic auditory nerve, malformed cochlea, or malfunctioning electrodes. Facial nerve stimulation is more common in patients with otosclerosis receiving $\mathrm{CI}$ as the new soft and remodeled bones have lower impedance. Facial nerve stimulation causes severe discomfort for the patients and their families because of the twitches of the facial muscles specially the lid muscles leading to eye closure with sounds. It is frightening to the parents in case of children. Functionally, facial nerve stimulation degrades the performance of CI users and badly affects their speech perception.

\section{Management of FNS}

Facial nerve stimulation can frequently be resolved with changes in some parameters in the audio- processor fitting but, in some cases, this can lead to a reduction in the patient performance Berrettini et al. [6]. Three common clinical remedies to prevent unpleasant FNS caused by activation of certain electrodes are to expand their pulse phase duration, simply deactivate them or decrease the MCL Bahmer \& Baumann [7].

\section{Switch off effected electrodes}

In this solution the audiologist or the programmer searches for the electrodes that cause FNS and switch them off. However, if more than one electrode causes FNS, this will cause more and more electrodes to be switched off. The more the switched off electrodes, the worse the patient performance is. Moreover, switching of many electrodes will affect frequency resolution of the CI and will degrade the speech discrimination of the patients because each electrode supply electrical pulses to specific frequency region. At MED-EL implants, for example, it is recommended to keep at least eight electrodes out of twelve on for good patient performance.

\section{Increase phase duration}

It was found that the more the phase duration or the pulse width of the biphasic pulse, the less probability of FNS occurs. But the drawback of widening the pulse width is the decrease of pulse rate per second which will decrease the spectral resolution of the speech signals and degrade the speech discrimination of the patients.

\section{Decrease of MCL}

Of course, this is a straight forward solution, but it will dramatically affect the patient performance directly by not coding substantial segment of speech signals according to their loudness. Unfortunately, in some patients these methods do not provide sufficient FNS prevention and limits the benefits of CI.

\section{Objectives}

The main objective of the current study was to evaluate the effect of applying the triphasic pulse stimulation in minimizing or eliminating the FNS in CI recipients. Other objective was to determine whether applying the triphasic pulse stimulation requires change(s) in the fitting parameters of the audio-processor (specially the MCL) to get the best patients performance. Final objective was to determine the effect of applying the triphasic pulse stimulation on the patient performance as measured subjectively from the patient satisfaction and objectively by measuring the aided pure tone audiometry and aided speech discrimination.

\section{Material and Methods}

Table 1: Relevant Data of the $\mathrm{Cl}$ subjects included in the current study.

\begin{tabular}{|c|c|c|c|c|c|c|c|c|c|c|c|c|}
\hline & Age & Sex & Ear & Et. & Implant & Elect. & R.W. & Processor & $\begin{array}{c}\text { Age } \\
\text { at } \\
\text { Imp. }\end{array}$ & $\begin{array}{c}\text { CT } \\
\text { scan }\end{array}$ & $\begin{array}{c}\text { FN } \\
\text { Injury }\end{array}$ & $\begin{array}{c}\text { FNS } \\
\text { Channels }\end{array}$ \\
\hline S1 & 36 & M & R & Prog. HL & Sync. & Flex28 & + & Sonnet & 32 & Norm. & No & All \\
\hline S2 & 31 & M & L & $\begin{array}{c}\text { Post } \\
\text {-trumatic HL }\end{array}$ & Sync. & Flex28 & + & Sonnet & 28 & Norm. & No & All \\
\hline S3 & 23 & F & R & S.S.D & Sync. & Flex28 & + & Sonnet & 20 & Norm. & No & All \\
\hline S4 & 5 & M & R & Cong. & Sync. & Form24 & + & Sonnet & 3 & IP2 & Exposed & All \\
\hline S5 & 4 & M & L & Cong. & Sync. & Flex28 & + & Sonnet & 2 & Norm. & Exposed & All \\
\hline
\end{tabular}

Table 1 shows the relevant data of the subjects included in the current study. Five CI recipients were included to this study. Four of them were implanted by MED- EL Synchrony Flex28 and one subject was implanted with MED-EL Synchrony Form 24 and all the subjects use Sonnet audio processor the external part. They were four males and one female with age range of 


\section{Global Journal of Otolaryngology}

4 years to 36 years. Years of implantation ranged from 2 years to 4 years. Four subjects had bilateral profound SNHL and one subject had single sided deafness. Hearing loss was diagnosed in infancy in two subjects (congenital SNHL). One subject had bilateral progressive SNHL; one subject had post-traumatic sudden SNHL; one subject had sudden unilateral profound SNHL. The radiological findings were normal CT and MRI scans for all subjects except S4 has malformed cochlea IP2. All subjects were implanted by the same surgeon and by the same surgical technique. The $\mathrm{CI}$ electrodes were inserted via round window with no injury to the FN during the operation.

All subjects experienced FNS from second or third session after the switch on session. FNS was at all channels. CT scan was performed post-operative to check and confirm the electrode placement inside the cochlea. Table 2 shows different methods used and failed to complete elimination of FNS in all subjects before the application of triphasic pulse strategy. Methods included decrease MCL, deactivate some channels in one subject, and increase pulse width.
Table 2: Previous FNS Management before the application of triphasic pulse.

\begin{tabular}{|c|c|c|c|}
\hline & Decrease MCL & $\begin{array}{c}\text { Deactivated } \\
\text { Elect. }\end{array}$ & $\begin{array}{c}\text { Increase Phase } \\
\text { duration }\end{array}$ \\
\hline S1 & + & - & + \\
\hline S2 & + & - & + \\
\hline S3 & + & 11,12 & + \\
\hline S4 & + & - & + \\
\hline S5 & + & - & + \\
\hline
\end{tabular}

\section{Results}

The MCL, THR, other fitting parameters and audiological performance was registered before and after applying triphasic pulse stimulation. (Figures 12-16) show MCL, THR, PTA and SRT for the five subjects in the biphasic and triphasic pulse stimulation. Figure 17 shows the same data averaged among the five subjects. In all subjects, triphasic pulse stimulation eliminated FNS in all electrodes except in 2 electrodes in one subject. All subjects were satisfied with this strategy with improvement in both aided pure tone threshold and SRT.
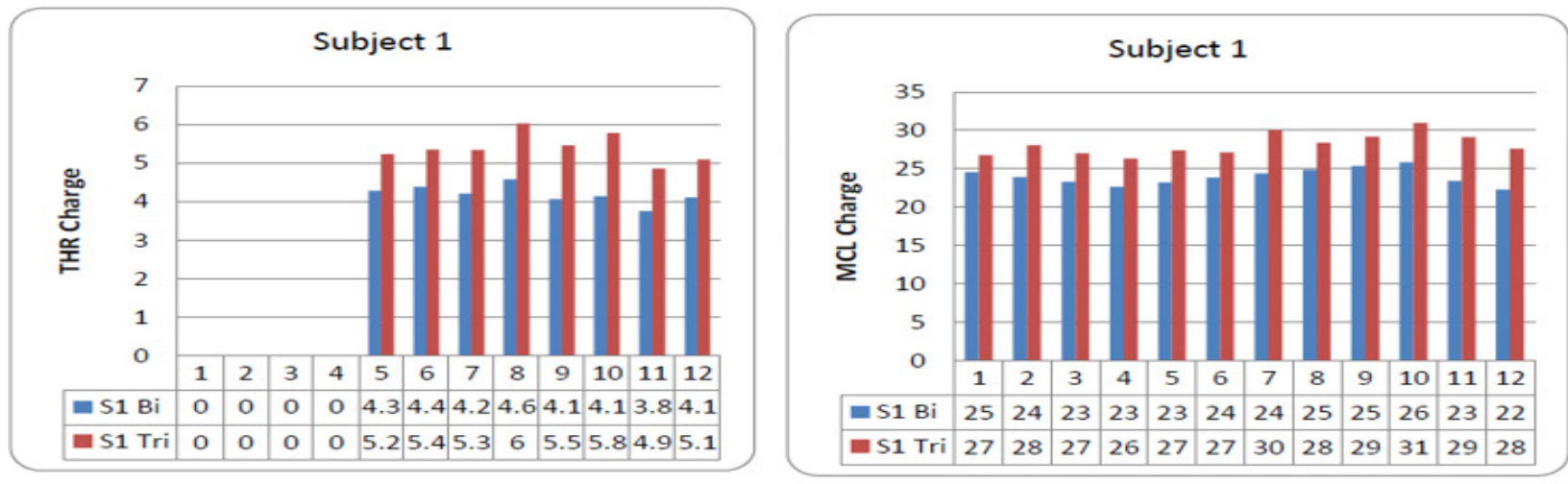

\section{Subject 1}

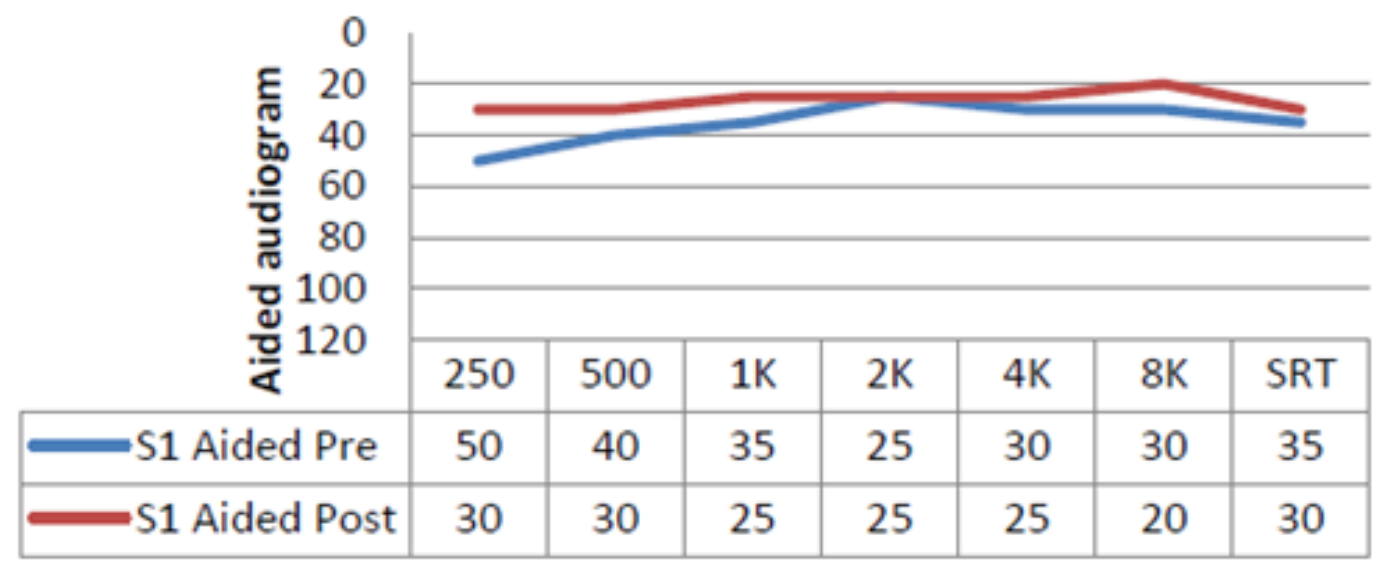

Figure 12: THR, MCL, Aided thresholds and SRT of subject 1. 

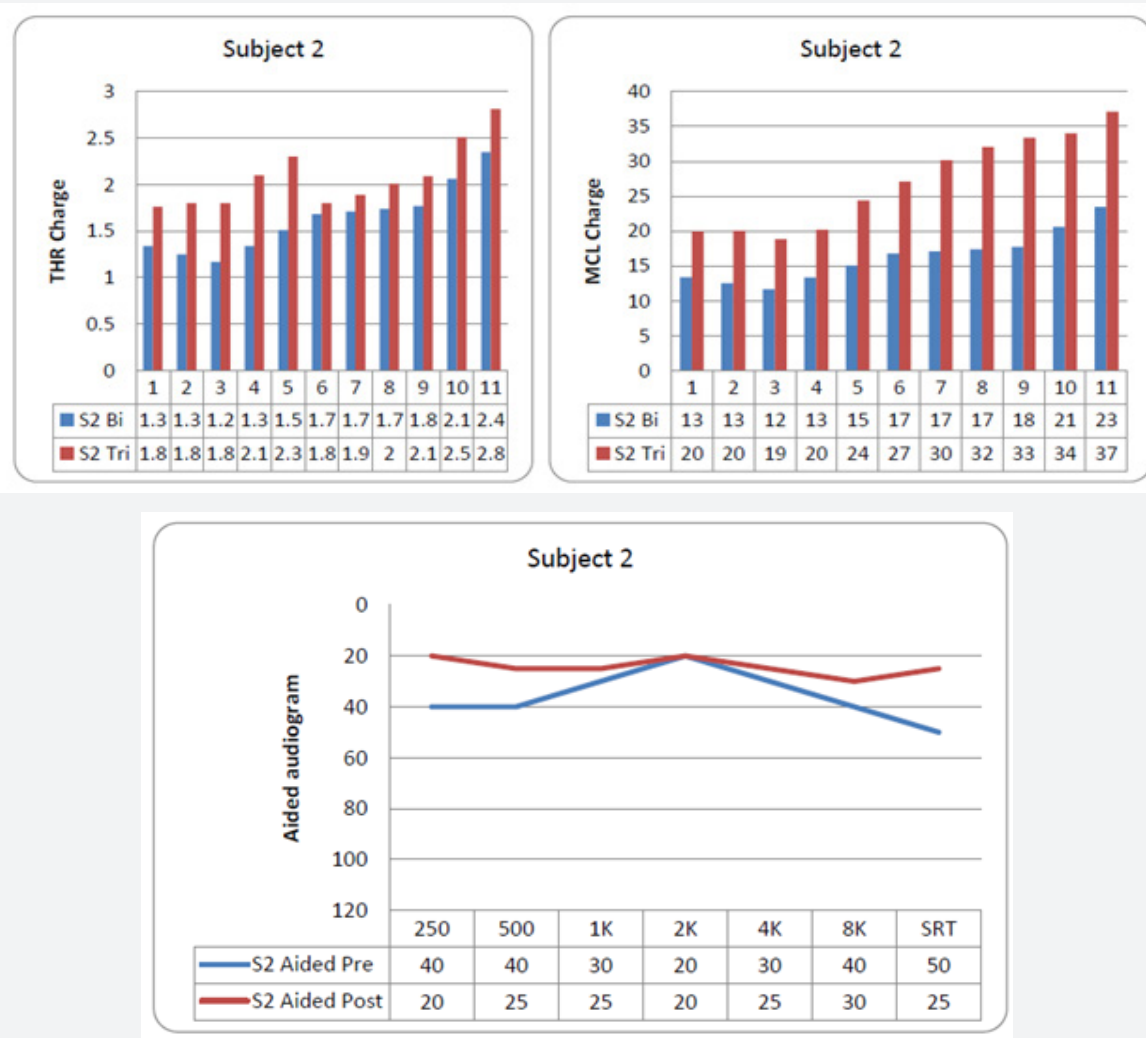

Figure 13: THR, MCL, Aided thresholds and SRT of subject 2.
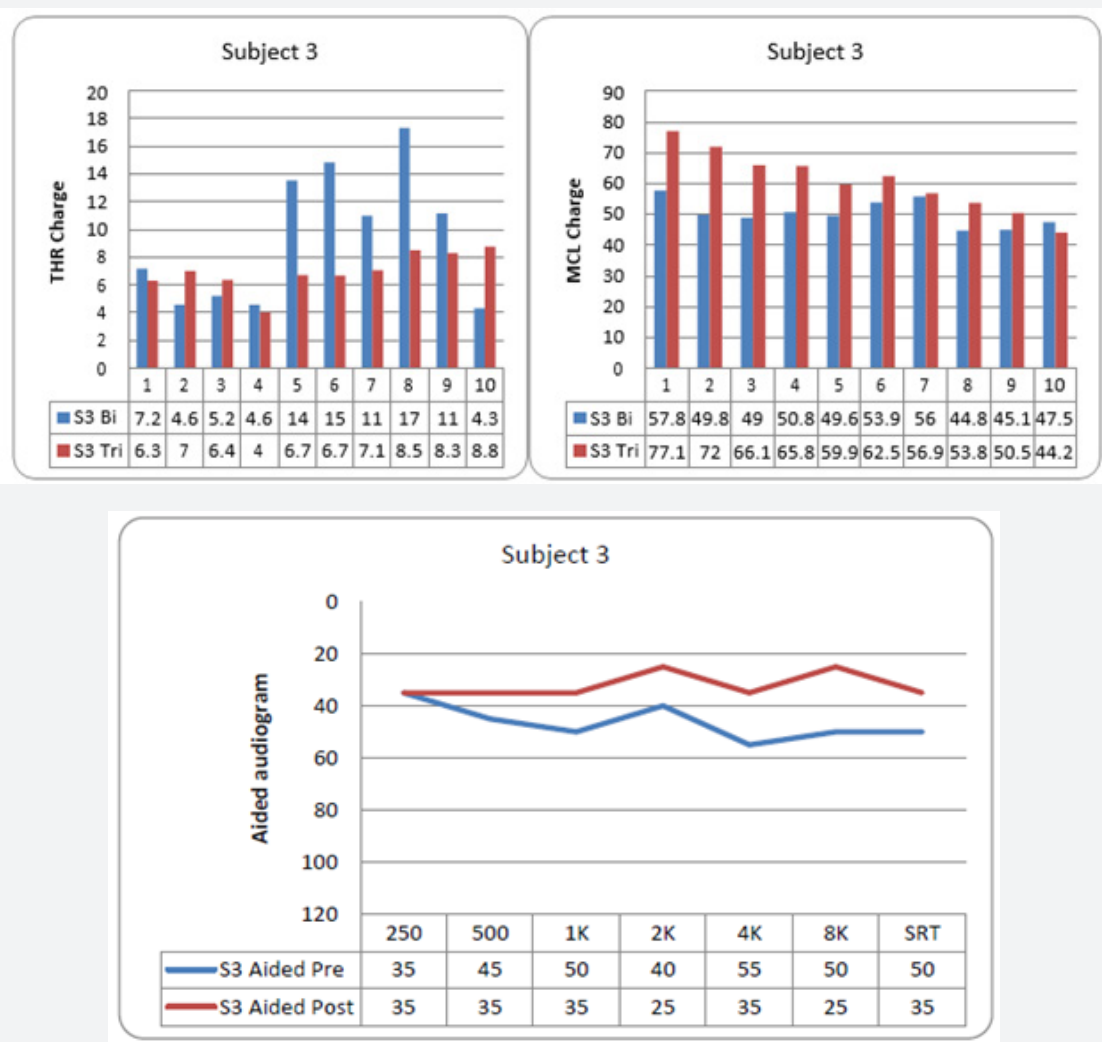

Figure 14: THR, MCL, Aided thresholds and SRT of subject 3. 

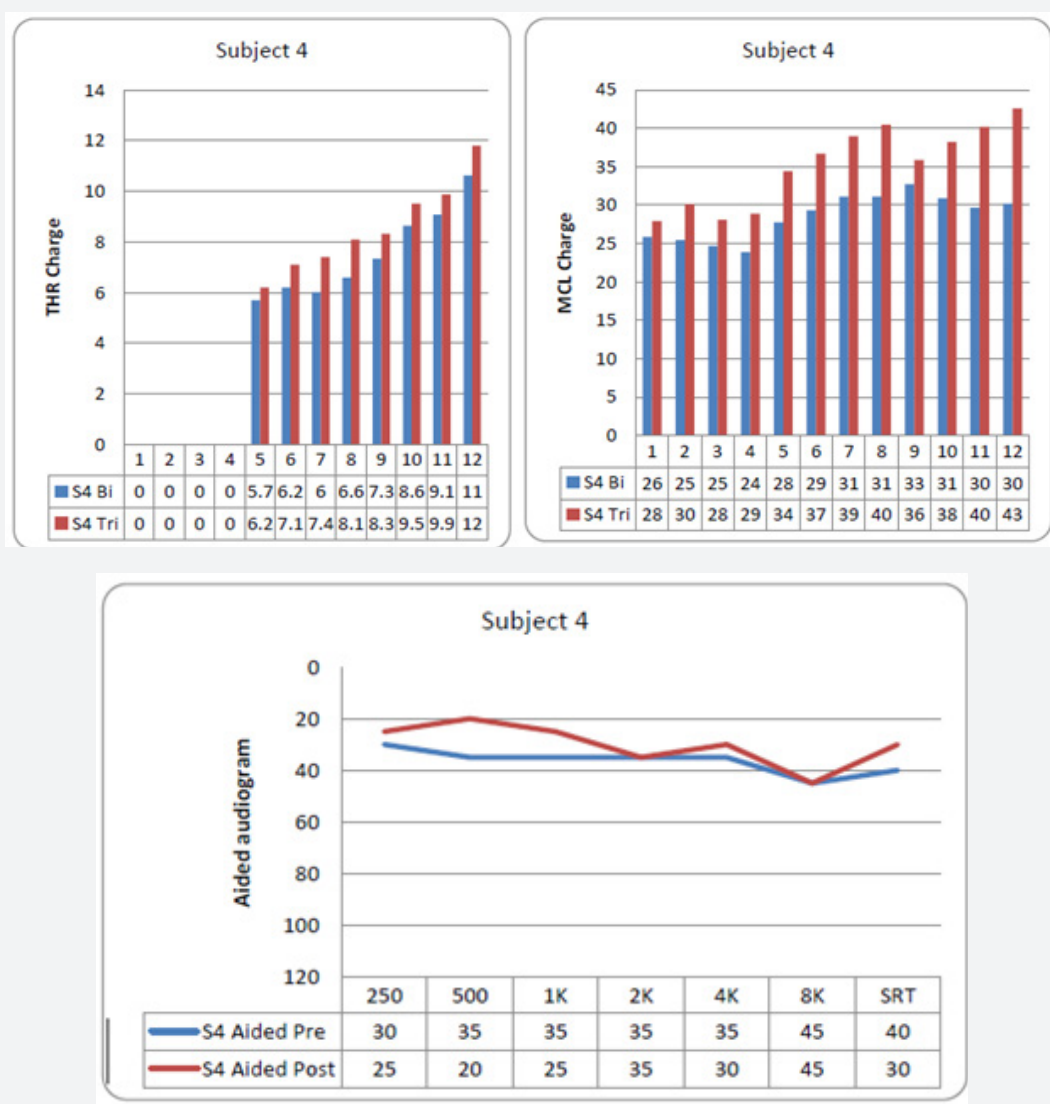

Figure 15: THR, MCL, Aided thresholds and SRT of subject 4.
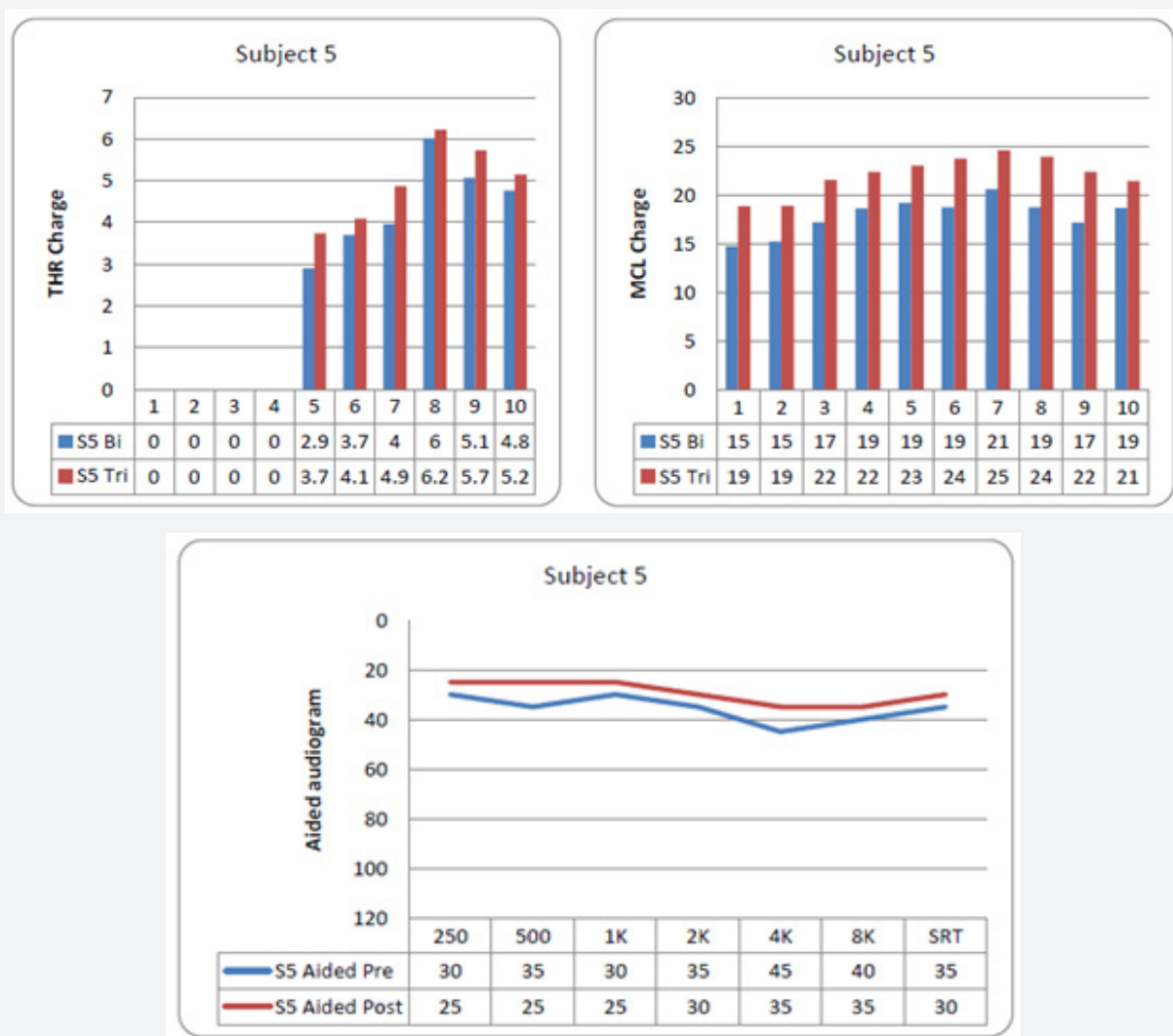

Figure 16: THR, MCL, Aided thresholds and SRT of subject 5. 

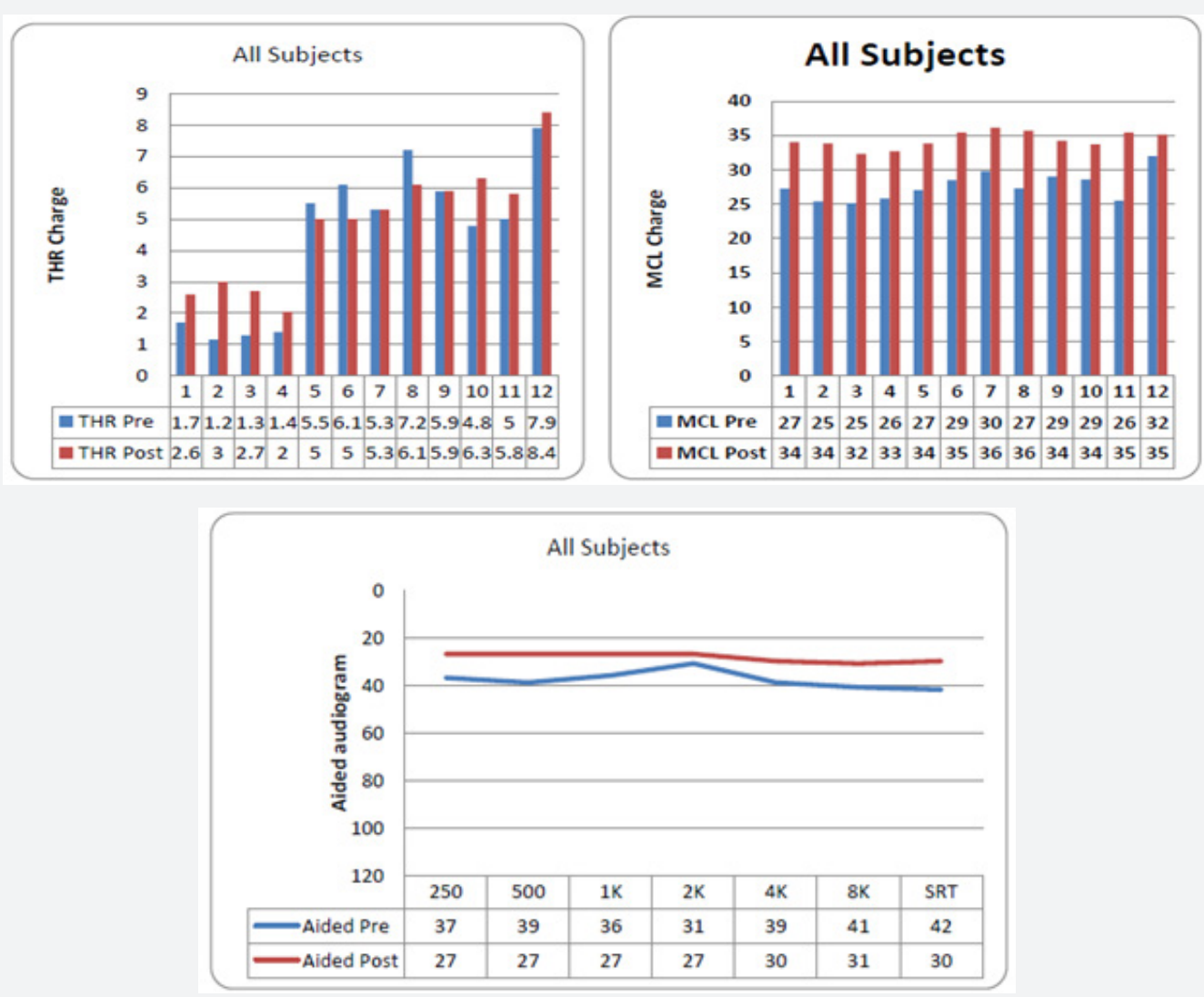

Figure 17: THR, MCL, Aided thresholds and SRT.

\section{Subject 1}

In this subject all electrodes were working and causing FNS. Figure 12 shows MCL, THR and aided audiogram before and after applying triphasic strategy are shown. Both MCL and THR had to be increased to achieve satisfactory map, which resulted in better aided pure tone threshold and better SRT (the SRT improved from was $35 \mathrm{~dB}$ to $30 \mathrm{~dB}$ ).

\section{Subject 2 (Figure 13)}

In this subject all electrodes were working except electrode twelve (it was found out of the cochlea). FNS was in all eleven electrodes. Similar to subject 1, MCL and THR had to be increased to achieve satisfactory map, which resulted in better aided pure tone threshold and better SRT (the SRT improved from was 50 $\mathrm{dB}$ to $25 \mathrm{~dB}$ ). In such subject, the improvement in the aided SRT was better than in subject 1 .

\section{Subject 4 (Figure 15)}

Subject 4 demonstrate same findings as previous subjects with elimination of the FNS and increase in both MCL and THR to achieve satisfactory maps. Not like the pervious subjects the triphasic inter-pulse gap had to be increased to 20 microseconds (the default value is 2.1 microseconds). Also, the Maplaw compression was changed from 500 to 700 to reach the patient satisfaction. The first four THR values were set to zero. The SRT improved from $40 \mathrm{~dB}$ to $30 \mathrm{~dB}$.

\section{Subject 5 (Figure 16)}

In this subject all electrodes were working except electrodes eleven and twelve they showed no response (they were switched off). Similar to subject 4, triphasic inter-pulse gap was increased to 20 microseconds (it was 2.1 microseconds), and the Maplaw compression was changed from 500 to 700 to reach the patient satisfaction. The first four THR values were set to zero. Both aided pure tone and SRT was improved after the triphasic pulse stimulation. The SRT improved from $35 \mathrm{~dB}$ to $30 \mathrm{~dB}$.

\section{Average of the 5 subjects (Figure 17)}

In average, the MCL values had to be increased from 5-10 units (across electrodes) with triphasic strategy the satisfactory maps. The THR values vary between both strategies. It is clear that with using the triphasic strategy the aided thresholds and the SRTs become better. Aided thresholds improved by average of $10 \mathrm{~dB}$ and also the SRT improved by more than $10 \mathrm{~dB}$. In addition, inter-pulse gab might be increased to 20 , and the compression value to 700 (found in two subjects but need more investigations and more subjects).

\section{Discussion}

The benefits of CI for patients who suffer from SNHL bilaterally or even unilaterally are outstanding. But, sometimes the fitting of these patients has drawbacks. One of these 
drawbacks is FNS. It is a very disturbing problem and limits the usage and benefits of CI. Triphasic stimulation pulses strategy was invented by MED-EL to eliminate or minimize FNS. The objectives of the current study were to test the effect of triphasic strategy in eliminating FNS and to determine the required change in fitting parameters and patient performance after applying the strategy. Results showed that triphasic stimulation eliminated FNS in all subjects and in all electrodes except in two electrodes in one subject. Moreover, patient's performance was better in triphasic strategy than in the traditional biphasic strategy. All subjects were satisfied and had better aided pure tone threshold and SRT. The MCL had to be increased to achieve patient satisfaction and the optimal maps. Such increase in the MCL might increase power consumption and might lead to change the power source more than biphasic strategy (needs more investigations). It is mandatory to repeat the current study in much larger sample. If same results were obtained, then it is highly recommended to use the triphasic pulse stimulation in cases of FNS as the first line of management to eliminate FNS as it gives better performance than the other methods to eliminate FNS as decrease MCL, increase pulse width, and deactivation of the channels. A disadvantage of triphasic strategy is that the fitting parameters (especially MCL and THR) must be remeasured to achieve the optimal maps and patient satisfaction. Another point that deserves study with triphasic stimulation is the need to increase in the inter-pulse gap in some patients to achieve the patient satisfaction. Increase the inter-pulse gap might decrease the pulse rate and may have a direct impact on speech discrimination [8].

\section{Conclusion}

Triphasic pulse stimulation is highly effective way to eliminate FNS in CI users with better patient performance than the traditional methods. Actually, this method should be the first option or solution the audiologist should pick up with patients who have FNS. But the audiologist has to take into account the modifications that should be done to the fitting map parameters. The MCL values are higher in triphasic maps compared to biphasic ones. This study should be performed on a larger scale and should take into account the other triphasic parameters like inter-pulse gap and precise triphasic pulses.

\section{References}

1. Mudry, Mills (2013) The early history of the cochlear implant: a retrospective. JAMA Otolaryngol Head Neck Surg 139(5): 446-453.

2. Kamal (2017) Comparison Between Behavioral, Modified Brown ECAP, ESRTComparison Between Behavioral, Modified Brown ECAP, ESRT. Juniper Publishers Inc.

3. MED-EL (2018) Austria.

4. Gifford René (2011) Who is a cochlear implant candidate? The Hearing Journal 64(6): 16-22.

5. Shapiro, Bradham (2012) Cochlear implant programming. Otolaryngol Clin North Am 45(1): 111-127.

6. Berrettini, Vito, Bruschini, Passetti, Forli (2011) Facial nerve stimulation after cochlear implantation: our experience. Acta Otorhinolaryngol Ital 31(1): 11-16.

7. Bahmer, Baumann (2016) The Underlying Mechanism of Preventing Facial Nerve Stimulation by Triphasic Pulse Stimulation in Cochlear Implant Users Assessed with Objective Measure. Otol Neurotol 37(9): 1231-1237.

8. Niparko (2010) STEPS TO A COCHLEAR IMPLANT.

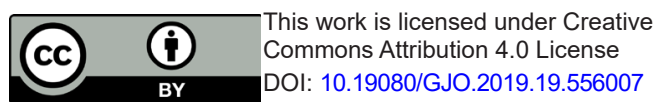

\section{Your next submission with Juniper Publishers will reach you the below assets}

- Quality Editorial service

- Swift Peer Review

- Reprints availability

- E-prints Service

- Manuscript Podcast for convenient understanding

- Global attainment for your research

- Manuscript accessibility in different formats

( Pdf, E-pub, Full Text, Audio)

- Unceasing customer service

Track the below URL for one-step submission https://juniperpublishers.com/online-submission.php 